\title{
LONGITUDINAL ELASTIC WAVES IN CYLINDERS AND TUBES-INCLUDING THERMOELASTIC EFFECTS
}

\author{
by F. J. LOCKETT \\ (Received 1st December 1958)
}

\section{Introduction}

Longitudinal elastic waves in solid circular cylinders were first investigated by Pochhammer (1) and Chree (2) but they did not take into account the effects that thermal properties have on the propagation of these waves. In this paper we shall consider waves in solid and hollow cylinders as well as in the infinite medium with a cylindrical cavity, and in each case we shall take account of the thermoelastic effects.

The type of wave considered can be characterised in cylindrical polar co-ordinates by the components of displacement

$$
u_{r}=R(r) e^{i(q z+p t)}, u_{z}=Z(r) e^{i(q z+p t)}, u_{\phi}=0 .
$$

Here the $z$-axis is the axis of the cylinder and we consider wave propagation with cylindrical symmetry. $R$ and $Z$ are functions of the radial coordinate $r$ only, and the problem is to find expressions for them such that the field equations and the boundary conditions are satisfied. In all the problems considered here the boundary conditions are such that the components of stress $\sigma_{r}$ and $\tau_{r z}$ vanish on the cylindrical boundaries. There is also a thermal radiation condition.

* The field equations for thermoelasticity have been given by Biot (3). We shall use a particular dimensionless form of these equations due to Chadwick and Sneddon (4).

If $\rho$ is the density of the medium, $c$ its specific heat at constant strain, $v_{P}$ and $v_{S}$ are the velocities of $P$-and $S$-waves, $\lambda$ and $\mu$ are Lamé's constants, $k$ is the thermal conductivity, $\alpha$ the coefficient of linear expansion, $\gamma=\alpha(3 \lambda+2 \mu)$, and $T$ is the absolute temperature of the medium in its reference state of zero stress and strain, then we can define a frequency

$$
\omega^{*}=\rho c v_{P}^{2} / k
$$

and take the quantities $1 / \omega^{*}, v_{P} / \omega^{*}, T$ and $\mu$ as our units of time, length, temperature and stress respectively. The field equations can then be written in terms of the dimensionless forms of the displacement vector $u$ and the temperature distribution $\theta$ as

where $\omega=\frac{1}{2} \operatorname{curl} \mathbf{u}$,

$$
\left.\begin{array}{c}
\beta^{2} \operatorname{grad} \operatorname{div} \mathbf{u}-2 \operatorname{curl} \omega-b \operatorname{grad} \theta=\beta^{2} \frac{\partial^{2} \mathbf{u}}{\partial t^{2}}-\mathbf{F} \\
\nabla^{2} \theta=\frac{\partial \theta}{\partial t}+g \frac{\partial}{\partial t}(\operatorname{div} \mathbf{u})-\Psi
\end{array}\right\}
$$

$$
\beta=v_{P} / v_{S}, b=\gamma T / \mu, g=\gamma /(\rho c)
$$


and $\mathbf{F}$ and $\Psi$ are the dimensionless forms of the body forces and heat sources respectively.

Since all frequencies obtainable in practice are much smaller than $\omega^{*}$, the main advantage of this system of units is that all frequencies obey the inequality $\omega \ll 1$. This fact would be extremely useful in an approximate numerical analysis of the results.

In an analysis where thermal effects are ignored it is obvious that equations (3) are replaced by the simpler vector equation

$$
\beta^{2} \operatorname{grad} \operatorname{div} \mathbf{u}-2 \operatorname{curl} \omega=\beta^{2} \frac{\partial^{2} \mathbf{u}}{\partial t^{2}}-\mathbf{F} .
$$

In the actual problems considered there are no body forces or heat sources present. However, we need to retain them in equations (3) and (5) to enable us to apply the method of solution given by Lockett (5), which appears as a companion paper in this issue.

\section{Non-Thermal Analyses}

(1) Solid Gircular Gylinder. The results of Pochhammer (1) and Chree (2) for this problem are well known. However, we shall re-derive their results here, since it illustrates the method on a relatively simple example whose result is already known, and because the results of the other two problems are quickly obtainable from this analysis.

Following the method of the companion paper, we consider the infinite medium subjected to wave propagation of the type (1) and to the action of body forces concentrated on the radius $r=d$, where $d \geqslant a$. We write these body forces in the form

$$
F_{r}=A d^{-1} \delta(r-d) e^{i(q z+p t)}, F_{z}=B d^{-1} \delta(r-d) e^{i(q z+p t)}
$$

where $\delta(x)$ is the Dirac delta function and $A$ and $B$ are to be chosen so that the components of stress $\sigma_{r}$ and $\tau_{r_{z}}$ should vanish on $r=a$. The exponential dependence is necessitated by the choice of the expressions (1) and the term $d^{-1}$ is put in for convenience at a later stage.

Substitution from (1) and (6) into (5) gives us the set of differential equations

$$
\left.\begin{array}{r}
\beta^{2}\left(R^{\prime \prime}+\frac{1}{r} R^{\prime}-\frac{1}{r^{2}} R+i q Z^{\prime}\right)+i q\left(i q R-Z^{\prime}\right)=-\beta^{2} p^{2} R-A d^{-1} \delta(r-d) \\
\beta^{2} i q\left(R^{\prime}+\frac{1}{r} R+i q Z\right)-\frac{1}{r}\left(i q R+i q R^{\prime}-Z^{\prime}-r Z^{\prime \prime}\right)=-\beta^{2} p^{2} Z-B d^{-1} \delta(r-d)
\end{array}\right\}
$$

which can be solved by the transform method.

Defining the Hankel transforms

$$
\bar{R}(\xi)=\int_{0}^{\infty} r J_{1}(\xi r) R(r) d r, \bar{Z}(\xi)=\int_{0}^{\infty} r J_{0}(\xi r) Z(r) d r
$$


equations (7) may be transformed and simplified to read

$$
\left.\begin{array}{l}
\left(\beta^{2} p^{2}-\beta^{2} \xi^{2}-q^{2}\right) \bar{R}-\left(\beta^{2}-1\right) i q \xi \bar{Z}=-A J_{1}(\xi d) \\
\left(\beta^{2}-1\right) i q \xi \bar{R}+\left(\beta^{2} p^{2}-\beta^{2} q^{2}-\xi^{2}\right) \bar{Z}=-B J_{0}(\xi d)
\end{array}\right\} .
$$

The solutions of these algebraic equations can be expressed in the form

where

$$
\begin{aligned}
\bar{R} & =-\beta^{-2} p^{-2} i q B \xi J_{0}(\xi d)\left\{\left(\xi^{2}+k_{1}^{2}\right)^{-1}-\left(\xi^{2}+k_{2}^{2}\right)^{-1}\right\} \\
& -\beta^{-2} A J_{1}(\xi d)\left\{-p^{-2} q^{2}\left(\xi^{2}+k_{1}^{2}\right)^{-1}+p^{-2} k_{2}^{2}\left(\xi^{2}+k_{2}^{2}\right)^{-1}\right\} \\
\bar{Z}= & -\beta^{-2} B J_{0}(\xi d)\left\{p^{-2} k_{1}^{2}\left(\xi^{2}+k_{1}^{2}\right)^{-1}-p^{-2} q^{2}\left(\xi^{2}+k_{2}^{2}\right)^{-1}\right\} \\
& +\beta^{-2} p^{-2} i q A \xi J_{1}(\xi d)\left\{\left(\xi^{2}+k_{1}^{2}\right)^{-1}-\left(\xi^{2}+k_{2}^{2}\right)^{-1}\right\}
\end{aligned}
$$

$$
k_{1}^{2}=q^{2}-\beta^{2} p^{2}, k_{2}^{2}=q^{2}-p^{2}
$$

and these expressions are in a convenient form for applying the inverse transforms

$$
R=\int_{0}^{\infty} \xi \bar{R} J_{1}(\xi r) d \xi, Z=\int_{0}^{\infty} \xi \bar{Z} J_{0}(\xi r) d \xi
$$

If we use the notation

$$
I_{m n}=I_{m}\left(k_{n} r\right), K_{m n}=K_{m}\left(k_{n} d\right)
$$

and re-group the terms in the resulting expressions, these integrations give

$$
\left.\begin{array}{l}
R=-\beta^{-2} p^{-2}\left\{\left(k_{2}^{2} A K_{12}+i q B k_{2} K_{02}\right) I_{12}-\left(A q^{2} K_{11}+i q B k_{1} K_{01}\right) I_{11}\right\} \\
Z=-\beta^{-2} p^{-2}\left\{\left(i q k_{2} A K_{12}-q^{2} B K_{02}\right) I_{02}-\left(i q A k_{1} K_{11}-B k_{1}^{2} K_{01}\right) I_{01}\right\}
\end{array}\right\} .
$$

The required integrals can be found in Erdélyi (6), p. 49, and have been evaluated for $0<r<d$. Thus it is at this stage that the physical requirement (that the body forces should be outside or on the radius $r=a$ ) enters the mathematics.

These forms for the integrals, as stated in the published tables, also require that $\mathscr{R} k_{1}>0$ and $\mathscr{R} k_{2}>0$. However, it can be verified that the results (16) satisfy the given conditions even when these conditions are not satisfied.

We can now introduce the parameters

and write

$$
\left.\begin{array}{rl}
L & =-\beta^{-2} p^{-2}\left(k_{2} A K_{12}+i q B K_{02}\right) \\
M & =-\beta^{-2} p^{-2}\left(i q A K_{11}-B k_{1} K_{01}\right)
\end{array}\right\}
$$

Since

$$
\left.\begin{array}{l}
R=k_{2} L I_{12}+i q M I_{11} \\
Z=i q L I_{02}-k_{1} M I_{01}
\end{array}\right\} \text {. }
$$

$$
\left.\begin{array}{c}
\sigma_{r}=\left(\beta^{2}-2\right)\left(\frac{\partial u_{r}}{\partial r}+\frac{u_{r}}{r}+\frac{\partial u_{z}}{\partial z}\right)+2 \frac{\partial u_{r}}{\partial r} \\
\tau_{r z}=\frac{\partial u_{r}}{\partial z}+\frac{\partial u_{z}}{\partial r}
\end{array}\right\}
$$

the expressions (16) and the boundary conditions, $\sigma_{r}=\tau_{r z}=0$ on $r=a$, imply that

E.M.S. -K 2

$$
\left.\begin{array}{rl}
2 i q k_{1} I_{11}^{\prime} L-\left[\left(\beta^{2}-2\right) p^{2} I_{02}-2 k_{2}^{2} I_{12}^{\prime}\right] M & =0 \\
\left(\beta^{2} p^{2}-2 q^{2}\right) I_{11} L+2 i q k_{2} I_{12} M & =0
\end{array}\right\}
$$


where the modified Bessel functions are now evaluated for $r=a$. These are the equations determining the ratio $L / M$. (The initial amplitude of the wave is arbitrary).

The compatibility of equations (18) leads to the frequency equation

$$
P \equiv\left|\begin{array}{ll}
2 i q k_{1} I_{1}^{\prime}\left(k_{1} a\right) & -\left(\beta^{2}-2\right) p^{2} I_{0}\left(k_{2} a\right)+2 k_{2}^{2} I_{1}^{\prime}\left(k_{2} a\right) \\
\left(\beta^{2} p^{2}-2 q^{2}\right) I_{1}\left(k_{1} a\right) & 2 i q k_{2} I_{1}\left(k_{2} a\right)
\end{array}\right|=0
$$

i.e.

$$
4 q^{2} k_{1} k_{2} I_{12} I_{11}^{\prime}=\left(\beta^{2} p^{2}-2 q^{2}\right) I_{11}\left[\left(\beta^{2}-2\right) p^{2} I_{02}-2 k_{2}^{2} I_{12}^{\prime}\right]
$$

which apart from slight differences of notation is the result obtained by previous authors (see e.g., Sneddon and Berry (7), Eq. (73.24)).

(2) Infinite medium with cylindrical cavity. The only difference between this and the previous analysis is that $d \leqslant a<r$ and we are therefore interested in evaluating the integrals (12) for $d<r$. It can be seen from the published tables that the result of this inequality is to reverse the roles of the modified Bessel functions $I$ and $K$ in the previous analysis. Thus $R$ and $Z$ are given by expressions of the form

$$
\left.\begin{array}{l}
\begin{array}{l}
R=k_{2} L K_{12}+i q M K_{11} \\
Z=i q L K_{02}-k_{1} M K_{01}
\end{array}
\end{array}\right\}
$$

and the frequency equation becomes

$$
Q \equiv\left|\begin{array}{ll}
2 i q k_{1} K_{1}^{\prime}\left(k_{1} a\right) & -\left(\beta^{2}-2\right) p^{2} K_{0}\left(k_{2} a\right)+2 k_{2}^{2} K_{1}^{\prime}\left(k_{2} a\right) \\
\left(\beta^{2} p^{2}-2 q^{2}\right) K_{1}\left(k_{1} a\right) & 2 i q k_{2} K_{1}\left(k_{2} a\right)
\end{array}\right|=0
$$

(3) Hollow circular cylinder. We now have to consider the extra body forces

$$
\begin{aligned}
& F_{r}=\left\{A_{1} d_{1}^{-1} \delta\left(r-d_{1}\right)+A_{2} d_{2}^{-1} \delta\left(r-d_{2}\right)\right\} e^{i(q z+p t)} \\
& F_{z}=\left\{B_{1} d_{1}^{-1} \delta\left(r-d_{1}\right)+B_{2} d_{2}^{-1} \delta\left(r-d_{2}\right)\right\} e^{i(q z+p t)}
\end{aligned}
$$

where

$$
d_{1} \geqslant a_{1}>a_{2} \geqslant d_{2}
$$

$a_{1}$ and $a_{2}$ being respectively the external and internal radii of the tube.

The components with suffix " 1 " behave like the forces in the solid cylinder analysis, and those with suffix " 2 " behave like the forces in the cavity analysis. It is easily seen that their combined effect leads to the expressions

$$
\left.\begin{array}{l}
R=k_{2} L_{1} I_{12}+i q M_{1} I_{11}+k_{2} L_{2} K_{12}+i q M_{2} K_{11} \\
Z=i q L_{1} I_{02}-k_{1} M_{1} I_{01}+i q L_{2} K_{02}-k_{1} M_{2} K_{01}
\end{array}\right\}
$$

We now have to apply the conditions $\sigma_{r}=\tau_{r z}=0$ on both $r=a_{1}$ and $r=a_{2}$, and this leads to a frequency equation

$$
\left|\begin{array}{ll}
P\left(a_{1}\right) & Q\left(a_{1}\right) \\
P\left(a_{2}\right) & Q\left(a_{2}\right)
\end{array}\right|=0
$$


where the minors $P$ and $Q$ are defined in (19) and (22), and the arguments $a_{1}$ and $a_{2}$ show that they are evaluated for $r=a_{1}$ and $\mathrm{r}=a_{2}$ respectively.

\section{Thermoelastic effects}

(1) Solid circular cylinder. To the forms (1) we now have to add the temperature distribution $\theta$ in the form

$$
\theta=\Theta(r) e^{i(q z+n t)} \text {. }
$$

Since there is an extra (thermal) condition to apply we have also to consider a concentrated heat source

$$
\Psi=C d^{-1} \delta(r-d) e^{i(q z+p t)}
$$

in addition to the body forces (6).

Substitution of (1), (6), (26) and (27) into (3) gives the set of differential equations

$$
\left.\begin{array}{l}
\beta^{2}\left(R^{\prime \prime}+\frac{1}{r} R^{\prime}-\frac{1}{r^{2}} R+i q Z^{\prime}\right)+i q\left(i q R-Z^{\prime}\right)-b \Theta^{\prime}=-\beta^{2} p^{2} R-A d^{-1} \delta(r-d) \\
\beta^{2} i q\left(R^{\prime}+\frac{1}{r} R+i q Z\right)-\frac{1}{r}\left(i q R+i q r R^{\prime}-Z^{\prime}-r Z^{\prime \prime}\right)-b i q \Theta=-\beta^{2} p^{2} Z-B d^{-1} \delta(r-d) \\
\Theta^{\prime \prime}+\frac{1}{r} \Theta^{\prime}-q^{2} \Theta=i p \Theta+g i p\left(R^{\prime}+\frac{1}{r} R+i q Z\right)-C d^{-1} \delta(r-d)
\end{array}\right)
$$

which are the thermal counterparts of equations (7).

We can now transform these equations to the form

$$
\left.\begin{array}{l}
\left(\beta^{2} p^{2}-\beta^{2} \xi^{2}-q^{2}\right) \bar{R}-\left(\beta^{2}-1\right) i q \xi \bar{Z}+b \xi \bar{\Theta}=-A J_{1}(\xi d) \\
\left(\beta^{2}-1\right) i q \xi \bar{R}+\left(\beta^{2} p^{2}-\beta^{2} q^{2}-\xi^{2}\right) \bar{Z}-b i q \bar{\Theta}=-B J_{0}(\xi d) \\
g i p \xi \bar{R}-g p q \bar{Z}+\left(\xi^{2}+q^{2}+i p\right) \bar{\Theta} \\
\operatorname{si}\left(J_{0}(\xi d)\right.
\end{array}\right\}
$$

where $\bar{R}$ and $\bar{Z}$ are defined by (8) and

$$
\bar{\Theta}(\xi)=\int_{0}^{\infty} r J_{0}(\xi r) \Theta(r) d r
$$

The solutions of equations (29) are found to be

$$
\begin{aligned}
& \bar{R}=\frac{\begin{array}{c}
D\left[\left(\xi^{2}+q^{2}+i p\right)\left(\xi^{2}+\beta^{2} q^{2}-\beta^{2} p^{2}\right)+i p b g q^{2}\right] \\
-i G_{1}\left[\left(\beta^{2}-1\right)\left(\xi^{2}+q^{2}+i p\right)+i p b g\right] \xi q-K\left[\xi^{2}+q^{2}-\beta^{2} p^{2}\right] b \xi
\end{array}}{\left(\xi^{2}+q^{2}-\beta^{2} p^{2}\right) \mathscr{D}} \\
& i D\left[\left(\beta^{2}-1\right)\left(\xi^{2}+q^{2}+i p\right)+i p b g\right] \xi q \\
& \left.\bar{Z}=\frac{+G\left[\left(\xi^{2}+q^{2}+i p\right)\left(\beta^{2} \xi^{2}+q^{2}-\beta^{2} p^{2}\right)+i p b g \xi^{2}\right]+i K\left[\xi^{2}+q^{2}-\beta^{2} p^{2}\right] b q}{\left(\xi^{2}+q^{2}-\beta^{2} p^{2}\right) \mathscr{D}}\right\} \\
& \bar{\Theta}=\frac{-D i p g \xi+G p g q-K\left(\beta^{2} \xi^{2}+\beta^{2} q^{2}-\beta^{2} p^{2}\right)}{\mathscr{D}}
\end{aligned}
$$

where

and

$$
\begin{aligned}
& D=A J_{1}(\xi d), \quad G=B J_{0}(\xi d), K=C J_{0}(\xi d) \\
& \mathscr{D}=\beta^{2}\left(\xi^{2}+q^{2}-p^{2}\right)\left(\xi^{2}+q^{2}+i p\right)+i p b g\left(\xi^{2}+q^{2}\right)
\end{aligned}
$$


We now write $\mathscr{D}$ in the form

$$
\mathscr{D}=\beta^{2}\left(\xi^{2}+k_{3}^{2}\right)\left(\xi^{2}+k_{4}^{2}\right)
$$

where $k_{3}^{2}$ and $k_{4}^{2}$ are the roots of the equation

$$
\beta^{2} x^{2}-\left(2 \beta^{2} q^{2}+i p \beta^{2}-\beta^{2} p^{2}+i p b g\right) x+\beta^{2}\left(q^{2}+i p\right)\left(q^{2}-p^{2}\right)+i p b g q^{2}=0 .
$$

It is then easily seen that

$\beta^{2} / \mathscr{D}=\left(k_{4}^{2}-k_{3}^{2}\right)^{-1}\left[\left(\xi^{2}+k_{3}^{2}\right)^{-1}-\left(\xi^{2}+k_{4}^{2}\right)^{-1}\right]$

$\beta^{2} \xi^{2} / \mathscr{D}=\left(k_{4}^{2}-k_{3}^{2}\right)^{-1}\left[-k_{3}^{2}\left(\xi^{2}+k_{3}^{2}\right)^{-1}+k_{4}^{2}\left(\xi^{2}+k_{4}^{2}\right)^{-1}\right]$

$\beta^{2} / \mathscr{D}\left(\xi^{2}+k_{1}^{2}\right)=X\left(\xi^{2}+k_{1}^{2}\right)^{-1}+Y\left(\xi^{2}+k_{3}^{2}\right)^{-1}+Z\left(\xi^{2}+k_{4}^{2}\right)^{-1}$

$\beta^{2} \xi^{2} / \mathscr{D}\left(\xi^{2}+k_{1}^{2}\right)=-k_{1}^{2} X\left(\xi^{2}+k_{1}^{2}\right)^{-1}-k_{3}^{2} Y\left(\xi^{2}+k_{3}^{2}\right)^{-1}-k_{4}^{2} Z\left(\xi^{2}+k_{4}^{2}\right)^{-1}$

$\beta^{2} \xi^{4} / \mathscr{D}\left(\xi^{2}+k_{1}^{2}\right)=k_{1}^{4} X\left(\xi^{2}+k_{1}^{2}\right)^{-1}+k_{3}^{4} Y\left(\xi^{2}+k_{3}^{2}\right)^{-1}+k_{4}^{4} Z\left(\xi^{2}+k_{4}^{2}\right)^{-1}$

where

$\left.X=\left[\left(k_{3}^{2}-k_{1}^{2}\right)\left(k_{4}^{2}-k_{1}^{2}\right)\right]^{-1}, Y=-\left[\left(k_{3}^{2}-k_{1}^{2}\right)\left(k_{4}^{2}-k_{3}^{2}\right)\right]^{-1}, Z=\left[\left(k_{4}^{2}-k_{1}^{2}\right)\left(k_{4}^{2}-k_{3}^{2}\right)\right]^{-1}.\right)$

Thus the expressions (31) can all be written as the sums of terms like

$$
Q \xi^{p}\left(\xi^{2}+k_{i}^{2}\right)^{-1} J_{\mu}(\xi d)
$$

where $Q$ is a coefficient not involving $\xi$, and where $p$ and $\mu$ can take the values 0 or 1 , and $i$ can take the value 1,3 or 4 .

If we now apply to the new forms of (31) the transforms inverse to (8) and (30) we find that $R, Z$ and $\Theta$ are the sums of terms of the type

$$
Q \int_{0}^{\infty} \xi^{p+1}\left(\xi^{2}+k_{i}^{2}\right)^{-1} J_{\mu}(\xi d) J_{v}(\xi r) d \xi
$$

which, when evaluated for $r<d$, is of the form

$$
\text { (coefficient) } \times K_{\mu}\left(k_{i} d\right) I_{\nu}\left(k_{i} r\right) .
$$

In each of the expressions for $R, Z$ and $\Theta$, we then collect together the terms involving the same Bessel function $I_{m}\left(k_{n} r\right)$, so that these expressions can be written in the form

$$
\text { (coefficient } \left.P_{m n}\right) \times I_{m}\left(k_{n} r\right) \text {. }
$$

Although we do not give the details of the algebra here, it is found that the coefficients $\boldsymbol{P}_{m n}$, which depend on $d$, can all be written down in terms of three new parameters $L, M$ and $N$. In so doing it is necessary to remember that $k_{3}^{2}$ and $k_{4}^{2}$ are roots of the equation (34). We then get

$$
\begin{aligned}
& R=i q L I_{11}+b k_{3} M I_{13}+b k_{4} N I_{14} \\
& Z=-k_{1} L I_{01}+i b q M I_{03}+i b q N I_{04} \\
& \Theta=\beta^{2}\left(k_{3}^{2}+p^{2}-q^{2}\right) M I_{03}+\beta^{2}\left(k_{4}^{2}+p^{2}-q^{2}\right) N I_{04}
\end{aligned}
$$

and it can be verified that these expressions satisfy (28) for $r<d$. 
If we now apply the boundary conditions $\sigma_{r}=0, \tau_{r z}=0, \partial \theta / \partial r+h \theta=0$, on $r=a$, we obtain three equations in $L, M$ and $N$ the compatibility of which demands that

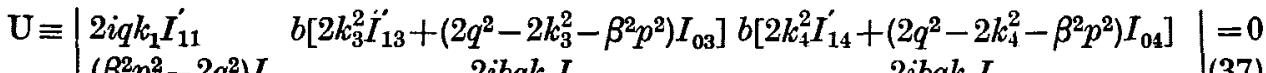

$$
\begin{aligned}
& \left(\beta^{2} p^{2}-2 q^{2}\right) I_{11} \quad 2 i b q k_{3} I_{13} \quad 2 i b q k_{4} I_{34} \\
& 0 \quad\left(q^{2}-p^{2}-k_{3}^{2}\right)\left(k_{3} I_{13}+h I_{03}\right) \quad\left(q^{2}-p^{2}-k_{4}^{2}\right)\left(k_{4} I_{14}+h I_{04}\right)
\end{aligned}
$$

where the modified Bessel functions are evaluated for $r=a$, i.e. $I_{m n}=I_{m}\left(k_{n} a\right)$. Equation (37) is the thermoelastic frequency equation.

(2) Infinite medium with cylindrical cavity. Having already derived the solutions to problems II and III from the solution of problem I for the non-thermal case, it is not necessary to describe how this is done for the thermal case. It is easily seen that the thermoelastic frequency equation is

$$
\mathrm{V} \equiv\left|\begin{array}{ccc}
2 i q k_{1} K_{11}^{\prime} & b\left[2 k_{3}^{2} K_{13}^{\prime}+\left(2 q^{2}-2 k_{3}^{2}-\beta^{2} p^{2}\right) K_{03}\right] & b\left[2 k_{4}^{2} K_{14}^{\prime}+\left(2 q^{2}-2 k_{4}^{2}-\beta^{2} p^{2}\right) K_{04}\right] \\
\left(\beta^{2} p^{2}-2 q^{2}\right) K_{11} & 2 i b q k_{3} K_{13} & 2 i b q k_{4} K_{14} \\
0 & \left(q^{2}-p^{2}-k_{3}^{2}\right)\left(k_{3} K_{13}+h K_{03}\right) & \left(q^{2}-p^{2}-k_{4}^{2}\right)\left(k_{4} K_{14}+h K_{04}\right)
\end{array}\right| \begin{gathered}
=0 . \\
(38)
\end{gathered} \mid
$$

(3) Hollow circular cylinder. From the results of problems I andIII and the reasoning given in the non-thermal theory we see that the new frequency equation is of the form

$$
\left|\begin{array}{ll}
U\left(a_{1}\right) & V\left(a_{1}\right) \\
U\left(a_{2}\right) & V\left(a_{2}\right)
\end{array}\right|=0
$$

where the minors $U$ and $V$ are defined in (37) and (38) and the arguments $a_{1}$ and $a_{2}$ indicate that the Bessel functions are evaluated for $r=a_{1}$ and $r=a_{2}$ respectively.

\section{Acknowledgments}

I am indebted to Professor I. N. Sneddon for suggesting this problem and to the Department of Scientific and Industrial Research for the award of a Research Studentship during the period in which the work was done.

\section{REFERENCES}

(1) L. Pochhammer, J. roine angew. Math., 81 (1876), 324.

(2) C. Chree, Q. J. Pure and Appl. Math., 23 (1889), 335.

(3) M. A. Biot, J. Appl. Phys., 27 (1956), 240.

(4) P. Chadwick and I. N. Sneddon, J. Mech. Phys. Solids, 6 (1958), 223.

(5) F. J. Lockett, Proc. Edin. Math. Soc., 11 (1959), 147-152.

(6) Erdélyi (Editor), Tables of Integral Transforms, Vol. II (McGraw-Hill Book Company) (1954).

(7) I. N. Sneddon and D. S. Berry, Handbuch der Physik, 6 (1958), 1.126 (Berlin, Springer-Verlag).

\section{Department of Mathematios}

The Untversity

GLasgow, W.2 